\title{
Correspondence
}

\section{Psychiatrists on Popper}

Sir: When a philosopher's work becomes as well known as that of Sir Karl Popper, it must be expected that it would be mis-quoted and mis-interpreted on occasions. Popper appears, however, to suffer more than most, often at the hands of psychiatrists. There have been two recent examples of this.

Taylor (Journal, July 1986, 149, 37-41) in his fascinating article on 'Hysteria, Play-acting and Courage', uses Popper's concepts of worlds I, II and III in support of his argument that hysteria is not a mental state but a product of human culture more akin to play-acting. In Popper's terms (Popper \& Eccles, 1981) this is therefore a world III phenomenon: world III being the universe of human artifacts such as novels, plays, and gardens. World III develops when consciousness or the human mind (world II) acts upon the material world (world I) to alter it in a meaningful way. Diagnosis, according to Taylor, is a world I phenomenon. This, I think, can be contested. Diagnosis is a concept and hence belongs either to world II or III. For example, in a disease (diagnosis) carcinoma of the lung, the world I phenomenon which relates to this diagnosis is the cancerous tissue itself, which, in itself, is not the diagnosis. That requires a mind (world I) before it can come into existence as a world III phenomenon. Hence, since all diagnoses/diseases are world III phenomena, the use of Popper's concepts in no way advances Taylor's argument that hysteria is different from other diagnoses, an argument which may nevertheless be correct.

The second example by Mathers (Bulletin, May 1986, 103-104) criticizes Popper's theory of science as it applies to psychiatry and, in particular, psychoanalysis. Popper's epistemology is a demonstration of the logical asymmetry between verification and falsification in the proof of theories. Mathers disagrees, preferring to believe in the possibility that falsifiability is not a prerequisite for testability and that theories can be proven to be true as well as to be false. In that case any theory which has been as decisively proven to be true as many have been proven false must be produced in evidence for this startling assertion. Of course many theories are held to be true by the scientific community because their falsifiability has been used to test them so frequently that a sort of Popperian truce has been called for the time being - but always with the possibility that an experiment at sometime in the future might disprove or modify the theory.

Mathers makes a further assumption which is attributed by implication to Popper, that non-science (psycho-analysis) is the equivalent of non-sense. Popper has pointed out that this is not the case. Indeed it would be difficult to devise an experiment to falsify Popper's own epistemological theory, but I doubt if he would accept that it is nonsense. Hence, Popper would not necessarily be led into asserting that analysis is non-sense only that it is non-science. Dr Mathers has therefore inadvertently performed for analysis more of a disservice than did Popper. CHRISTOPHER THOMPSON

Charing Cross Hospital and

Westminster Medical School London W6 8RF

\section{Reference}

POPPER, K. R. \& Eccles, J. C. (1981) The Self and Its Brain. New York: Springer International.

\section{Tardive Dyskinesia and Parkinsonism}

Sir: We read with interest the letter by Drs Oyebode \& McClelland (Journal, July 1986, 149, 122-123). Their results coincide with our clinical observations that coexistence of drug-induced parkinsonism (PS) and tardive dyskinesia (TD) in the same subjects is not exceptional, and that the intensity of the two disorders is not correlated. Nevertheless, we do not think that 'an independence of their relative severities calls into question the currently held view that PS results from a blockade and TD a hypersensitivity of dopamine receptors'. Nor do we consider that 'the two conditions are either mediated through different dopamine systems or through independent but related neurotransmitter systems'.

Easier explanations are available. Some nigrostriatal dopaminergic regions could be hypofunctioning as a result of the neuroleptic blockade of these neurons, while other regions could have become 'hypersensitivised' after a prolonged blockage at the same time. Differing levels of receptor sensitivity in the corpus striatum have been proposed by Carlsson (1970). Moreover, observations of parkinsonian tremor shifting into a dyskinetic movement would support the hypothesis that dopaminergic hyperfunction, clinically TD, would develop after 